Corollary. If $k=1$ and $* e_{0} x e_{\nu}^{2}=x$, then $* a x e_{0} b=* a e_{0} x b$ for any a and $b$.

ProOF.

$$
\begin{aligned}
* a x e_{0} b & \left.=* a * e_{0} e_{\nu} e_{\nu-1}^{2} x e_{0} b \quad \text { (by (2) with } m=1\right) \\
& =* a e_{0} * e_{\nu} e_{\nu-1}^{2} x e_{0} b \\
& =* a e_{0} x b \quad(\text { by Theorem } Q \text { with } m=1)
\end{aligned}
$$

UNIVERSITY OF BRISTOL

\title{
TWO THEOREMS ON FINITELY GENERATED GROUPS
}

\section{EUGENE SCHENKMAN}

Let $G$ be a group generated by a finite subgroup $H$ and an element $b$ of finite order. If $H$ commutes elementwise with $b$ (for this we shall write $[h, b]=e$ for every $h \in H$ where $[h, b]$ designates $\left.h b h^{-1} b^{-1}\right)$, then clearly $G$ is finite and $b$ is in the center of $G$.

We consider here the case where, for every $h \in H,[[h, b] b]=e$, and prove the following theorem:

Theorem. Let $G$ be generated by the finite subgroup $H$ and the element $b$ of finite order and, for every $h \in H$, let $[[h, b] b]=e$. Then $G$ is finite and $b$ is in the nil radical of $G$.

Proof. For $i=1,2, \cdots, n$ let $h_{i}$ be the elements of $H$. Then $h_{i}^{-1} b h_{i}$ are all the conjugates of $b$; for $b h^{-1} b h b^{-1}=h^{-1} b h$ by virtue of the hypothesis $[[h, b] b]=e$.

It follows from the fact that a finite set of conjugates generate a finite normal subgroup (cf. [1]) that $b$ is contained in a finite normal subgroup $K$ of $G$. But $H$ is finite and hence so also is $G / K$; and then finally $G$ is finite.

Furthermore since $b$ is in the center of $K, b$ is in the nil radical of $G$ as was asserted.

We can deduce another result from the fact that $[[g, b] b]=e$ for every $g \in G$ implies that $b$ is in the center of a normal subgroup of $G$.

THEOREM. Let $G$ be a finitely generated group with the property that if $b_{1}, \cdots, b_{n}$ are the generators of $G$, then $\left[\left[g, b_{i}\right] b_{i}\right]=e$ for every $g \in G$ and for $i=1,2, \cdots, n$. Then $G$ is nilpotent of class at most $n$. If furthermore the $b_{i}$ are of finite order then $G$ is finite.

Received by the editors September 13, 1953. 
Proof. For $i=1,2, \cdots, n$ let $B_{i}$ be the normal subgroup of $G$ in which $b_{i}$ is central. Since the $B_{i}$ are normal subgroups of $G$, so is each of the intersections $B_{i_{1}} \cap \cdots \cap B_{i_{r}}$ normal in $G$. For $j=1, \cdots$, $n$ let $A_{j}$ represent the subgroup of $G$ generated by the product of all possible intersections of $j$ of the $B_{i}$ at a time; i.e., $A_{1}=B_{1} B_{2} \cdots B_{n}$, $A_{2}=\left(B_{1} \cap B_{2}\right)\left(B_{1} \cap B_{3}\right) \cdots\left(B_{n-1} \cap B_{n}\right)$, etc., and $A_{n}=B_{1} \cap B_{2} \cap \cdots$ $\cap B_{n}$.

Then $A_{n}$ is in the center of $G$; for $A_{n}$ commutes elementwise with all the generators of $G$. And for each $r=1, \cdots, n, A_{r-1} / A_{r}$ is in the center of $G / A_{r}$. For each component $B_{i_{1}} \cap \cdots \cap B_{i_{r-1}}$ commutes elementwise with $b_{i_{1}}, \cdots, b_{i_{r-1}}$ and $\left(B_{i_{1}} \cap \cdots \cap B_{i_{r-1}}\right) \cap B_{i_{r}} \subset A_{r}$; hence modulo $A_{r}$ each component of $A_{r-1}$ is in the center of $G / A_{r}$ and consequently $A_{r-1} / A_{r}$ is in the center of $G / A_{r}$ as asserted. Hence $G$ is nilpotent of class at most $n$. The finiteness of $G$ follows immediately from this if the $b_{i}$ are of finite order.

COROLLARY. If $G$ is a finitely generated group all of whose elements have order 3, then $G$ is finite (cf. [2]).

For let $a$ and $b$ be any two elements of $G$. Then $[[b, a] a]$ $=b a b^{2} a b a^{2} b^{2} a^{2}=(b a b)(b a b)(b a b)\left(b^{2} a^{2}\right)\left(b^{2} a^{2}\right)\left(b^{2} a^{2}\right)=e$.

\section{BIBLIOGRAPHY}

1. A. P. Dietzmann, Einige Sätze über unendliche Gruppe Gedenkwerk D. A. Grave, Moscow 63-67 (1940) Russian.

2. W. Burnside, On an unsettled question in the theory of discontinuous groups, Quart. J. Math. vol. 33 (1902) pp. 230-238.

Louisiana State University and

INSTITUTE FOR ADVANCED STUDY 